\title{
Risk factors for dropped nucleus in cataract surgery as reflected by the European Registry for Quality Outcomes in Cataract and Refractive Surgery (EUREQUO)
}

Running head: Risk factors for dropped nucleus in cataract surgery

Mats Lundström, MD, $\mathrm{PhD}^{1}$, Mor Dickman, $\mathrm{MD}, \mathrm{PhD}^{2}$, Ype Henry, MD, FEBO ${ }^{3}$, Sonia Manning, MD, FRCSI (Ophth) ${ }^{4}$, Paul Rosen, FRCS, FRCOphth ${ }^{5}$, Marie-José Tassignon, MD, $\mathrm{PhD}, \mathrm{FEBO}^{6}$, David Young, $\mathrm{PhD}^{7}$, Ulf Stenevi, $\mathrm{MD}, \mathrm{PhD}^{8}$

${ }^{1}$ Department of Clinical Sciences, Ophthalmology, Faculty of Medicine, Lund University, Lund, Sweden; ${ }^{2}$ University Eye Clinic, Maastricht University Medical Centre, Maastricht, the Netherlands; ${ }^{3}$ Department of Ophthalmology, VUmc, Amsterdam, the Netherlands; ${ }^{4}$ Department of Ophthalmology, University Hospital Waterford, Waterford, Ireland; ${ }^{5}$ Oxford Eye Hospital, Oxford, United Kingdom; ${ }^{6}$ Department of Ophthalmology, Antwerp University Hospital, University of Antwerp, Antwerp, Belgium; ${ }^{7}$ Department of Mathematics and Statistics, University of Strathclyde, Glasgow, United Kingdom; ${ }^{8}$ Department of Ophthalmology, Sahlgrenska University Hospital, Mölndal, Sweden.

This study was supported by grants from the European Society of Cataract and Refractive Surgeons. No author has any financial interest in the materials or methods described in this study. 
Correspondence: Mats Lundström, Trossögatan 4, 37137 Karlskrona, Sweden. E-mail: mats.lundstrom@karlskrona.mail.telia.com

\footnotetext{
Abstract

Purpose: To analyse the incidence, risk factors, and outcomes of cataract surgery complicated by a dropped nucleus.

Setting: Patients who have received cataract surgery in 18 European countries.

Design: Retrospective cross-sectional register-based study.

Methods: Data from the European Registry for Quality Outcomes in Cataract and Refractive Surgery (EUREQUO) were analysed. The EUREQUO contains preoperative baseline, intraoperative, and follow-up data. Intraoperative data include dropped nucleus as a complication. Baseline data such as demographic data, ocular comorbidities, surgical difficulties, and visual and refractive outcomes were tested for association with dropped nucleus for the study period 1 January 2008 to 31 December 2018.

Results: The number of reported surgeries with complete data was 1,715,348. Dropped nucleus was reported in 1,221 $(0.071 \%)$ cases during the study period. White cataract, previous vitrectomy, poor preoperative visual acuity, small pupil, pseudoexfoliation, diabetic retinopathy, and male gender were significantly related to dropped nucleus. Year of surgery showed a significant trend of decreasing occurrence of dropped nucleus over time. Eyes with the complication of a dropped nucleus had a poorer visual and refractive outcome also compared with eyes with existing risk factors but no such complication.

Conclusion: Many risk factors for dropped nucleus were identified. A significant trend of decreasing occurrence of dropped nucleus was found for the study period. The visual and refractive outcome was poorer for eyes with dropped nucleus.
} 


\section{Introduction}

A clinical registry collecting a well-defined and large prospective data set is particularly suitable for the identification and analysis of rare events and complications and their outcomes. This has previously been shown for postoperative endophthalmitis by the Swedish National Cataract Register ${ }^{1}$ and for cataract surgery outcomes in eyes with previous corneal refractive surgery by the European Register for Quality Outcomes in Cataract and Refractive Surgery (EUREQUO). ${ }^{2}$ The EUREQUO ${ }^{3}$ was instituted in 2008 as a co-project between the European Union and the European Society of Cataract and Refractive Surgeons (ESCRS). The list of parameters includes surgical complications such as dropped nucleus, one of the most feared complications of modern cataract surgery. In this study, we will analyse the risk factors for dropped nucleus and the incidence of this complication as reflected by the EUREQUO. The surgical outcome will also be described.

\section{Methods}

The EUREQUO registry consists of two parts - cataract surgery and refractive surgery - on the same web-based platform. In this study, we used data from the cataract surgery register. The register contains data on baseline characteristics, surgery, and follow-up. The web form guidelines for this database describe mandatory baseline data to be reported, including year of birth, gender, eye, best corrected distance visual acuity, target refraction, co-existing eye diseases, surgical difficulties, and previous ophthalmic surgeries. Mandatory surgical data to be reported include date of surgery, type of operation, intraocular lens (IOL) material, and complications during surgery. Under "Surgical complications", report options are "none", "posterior capsule rupture", "vitreous loss", “dropped nucleus", “iris damage”, and "other". Complete reporting also includes mandatory follow-up data, e.g. date of examination, uncorrected distance visual acuity, best corrected distance visual acuity, refraction, macular degeneration (known before or revealed after cataract extraction), and postoperative complications. Clinics decide before joining the database whether they will report complete data or only preoperative and surgical data. This agreement between a clinic or surgeon and the EUREQUO gives access to the report function with a login page. 
Coding guidelines explain how data should be reported. "Surgical difficulties" include "small pupil", meaning that enlargement/stabilization of the pupil was produced using mechanical stretching devices. "White pupil" should be ticked if capsular staining has been used because of white pupil or dense brown cataract obscuring the red reflex. "Dropped nucleus" refers to either a complete nucleus or large nucleus pieces that have dropped, but not small lens cortex fragments.

In accordance with the protocol, consecutive cases were reported to the database by participating units, and a follow-up examination was performed 7-60 days after surgery. The total number of cataract extractions reported to the EUREQUO until 14 June 2019 was $2,865,897$.

All data reported to the EUREQUO are anonymised. This study was performed according to the tenets of the Declaration of Helsinki.

$\underline{\text { Statistical methods }}$

Factors associated with dropped nucleus were investigated using logistic regression. Stepwise selection was then used to construct a multivariate logistic model with the factors that wer e significant in the univariate analysis. Complication rates between experienced and inexperi enced surgeons were compared using Z-tests for two proportions and postoperative visual and refractive outcomes were compared between groups using tests. All analyses were done using IBM SPSS version 25 (SPSS Inc., Chicago, IL, USA) at a $5 \%$ significance level.

\section{Patients and materials}

The total number of reported cataract extractions containing the parameter of surgical complications (including dropped nucleus), with follow-up data, is 1,715,348 for the study period of 1 January 2008 to 31 December 2018. The number of reporting clinics has varied between 92 and 139 per year. 


\section{Results}

The mean age of patients with complete data was 74 (SD 10) years. Fifty-eight per cent were female. During the study period, 1,221 cases of dropped nucleus were reported. This corresponds to a frequency of $0.071 \%$. Among these patients $48 \%$ were female and their mean age was 75 (SD 10) years. Table 1 shows the annual number of reported cataract extractions with dropped nucleus as a complication parameter and the reported number of dropped nucleus cases

Table 1 here.

The register contains information on type of surgery. In Table 2 the number of dropped nuclei per type of surgery is shown. In 4,461 procedures $(0.3 \%)$ data on type of surgery was missing.

Table 2 here.

Table 2 shows that the highest frequency of dropped nucleus occurred during phacoemulsification combined with a filtering procedure. The lowest frequency was found with extracapsular cataract extraction (ECCE).

Univariate regression analyses identified factors related to dropped nucleus (Table 3).

Table 3 here.

Multivariate stepwise logistic regression analysis was performed to determine the parameters associated with dropped nucleus, as shown in Table 4.

Table 4 here.

Table 4 shows that being male, poor preoperative visual acuity, previous vitrectomy, white cataract, small pupil, pseudoexfoliation, diabetic retinopathy and year of surgery were associated with dropped nucleus. With time, there was a significant decreasing trend of dropped nucleus by year of surgery $(\mathrm{p}<0.001)$.

White cataract combined with previous vitrectomy occurred in 439 cases reported during the study period. Among these cases, 15 (3.4\%) dropped nucleus complications occurred, compared with 1,206 $(0.1 \%)$ for the rest of the database. The odds ratio (OR) for the dropped nucleus complication when previous vitrectomy is combined with white cataract was 50.3 (95\% CI 29.9-84.4). If combinations of risk factors (e.g. previous vitrectomy + white cataract) are included in the stepwise regression analysis, they become excluded in the 
equation because of no significance when previous vitrectomy and white cataract are also included as independent variables.

The parameters in the EUREQUO database have recently been expanded to include surgeon experience. The number of reported surgeries with this parameter included was 35,467. Eighteen dropped nucleus complications were reported among these procedures. Eight $(0.79 \%)$ of these complications occurred in 1,015 surgeries performed by inexperienced surgeons (trainees), which is a significantly higher frequency than the ten $(0.03 \%)$ dropped nucleus complications that occurred in 34,452 surgeries among experienced surgeons.

\section{Visual and refractive outcomes}

The visual outcome after dropped nucleus was significantly worse compared with no dropped nucleus complication; postoperative mean logarithm of minimum angle of resolution ( $\log$ MAR) best corrected visual acuity (BCVA) 0.24 \pm 0.32 v $0.05 \pm 0.17$ (Decimal: 0.6 v 0.9) ( $p<0.001$ ). In $74.6 \%$ of cases with complications, the postoperative BCVA was logMAR 0.3 ( 0.5 or $20 / 40)$ or better, compared with $95.5 \%$ where no such complication occurred. The refractive outcome after a dropped nucleus complication was also significantly worse compared with no such complication (mean absolute biometry prediction error of $1.02 \mathrm{D}$ compared with $0.43 \mathrm{D}(\mathrm{p}<0.001))$.

A significant difference in visual and refractive outcomes was found also when the comparison was confined to cases with a certain risk factor (Table 5). The visual outcome in functional visual acuity groups according to Lundström et al. ${ }^{4}$ is described in Table 6 . The visual acuity groups represent meaningful visual acuity levels from the patients' perspective. ${ }^{4}$ Table 6 shows the visual outcome for 619 cases with dropped nucleus. The remaining number (602) lacked information on preoperative visual acuity or the visual outcome. Table 6 shows that among eyes with excellent or very good vision before surgery (groups $1+2)(n=48)$, eight $(16.7 \%)$ deteriorated to worse than driving vision (groups 4+5). On the other hand, among eyes with poor vision before surgery (group 5; $\mathrm{n}=189), 74(39.2 \%)$ achieved excellent or very good vision (groups 1+2) and another 44 (23.3\%) achieved at least driving vision (group 3). The visual outcome was related to risk factors. Patients with dropped nucleus and risk factors (white cataract, previous vitrectomy, etc.) had a worse visual outcome compared with patients 
with dropped nucleus without risk factors (mean postoperative BCVA, logMAR: 0.30 (SD $0.33)(0.5)$ v $0.17(\operatorname{SD} 0.28)(0.7))$.

The occurrence of reported postoperative complications was significantly higher for cases with dropped nucleus compared with no such complications (135 cases with postoperative complications out of 744 dropped nucleus with reported postoperative outcome data, $\mathrm{p}<0.001$, OR 10.9 (95\% CI 9.1-13.2)). The most frequently reported postoperative complication for cases with dropped nucleus was "other", 15.6\%. Other important postoperative complications were uncontrolled intraocular pressure (IOP) (9.6\%), corneal oedema (4.4\%) and endophthalmitis (3.0\%).

\section{Discussion}

Dropped nucleus is one of the most feared complications of modern cataract surgery. Published studies indicate ${ }^{5,6}$ that the incidence of dropped nucleus is low. However, any cataract surgeon with a reasonable case volume and a wide spectrum of case complexity will at some point experience this complication. Identifying the risk factors and combinations thereof preoperatively would allow for advance planning, counselling, and case selection. Once a nucleus drops, information on visual and refractive outcomes is important to address patient questions and concerns.

Several risk factors for dropped nucleus were found in our study. These included previous vitrectomy, white cataract, poor preoperative visual acuity, small pupil, diabetic retinopathy, male gender, and pseudoexfoliation. In a smaller cohort, having an inexperienced surgeon was also a risk factor. Advance planning for individual risk factors can potentially increase safety. For example, a variety of techniques are available to manage a small pupil. Identifying a small pupil preoperatively and ensuring the necessary equipment is available in the operating room can prevent complications. Moreover, our study confirms that risk factors summate. In extremely high-risk cases, it is important that surgery is justified and that the patient is appropriately informed. Aasuri et al. found inexperienced surgeons, previous vitrectomy and, "total" cataract to be risk factors for dropped nucleus. ${ }^{7}$ They also found that topical anaesthesia could be a risk factor. However, we cannot confirm the latter in our study (data not shown). 
Dropped nucleus on average occurred in $0.071 \%$ of all reported cataract extractions, with a decreasing frequency over time. This is a lower figure than reported by Clark et al. ${ }^{5}$ who found a frequency of $0.12 \%$ during 22 years in Australia. Their study, however, ended in 2001. Moreover, they reported a decline in incidence of dropped nucleus with phacoemulsification technique over time, which corresponds to our findings, though we had a lower average. From the Netherlands, a frequency of $0.09 \%$ of retained lens fragments in the vitreous has been reported after phacoemulsification. ${ }^{6}$ Including retained lens fragments is a wider approach than focusing on complete dropped nuclei; furthermore, that study was published in 1997, which means earlier in the phacoemulsification era than our study. The chosen operation method, "type of surgery", in the register may have affected the trend of decreasing frequency of dropped nucleus in our study. With ECCE, dropped nucleus is less common given a posterior-to-anterior pressure gradient directing the lens forward, and no intra-lenticular instrumentation. By contrast, fluid infusion in phacoemulsification maintains anterior pressure in the presence of a practically sealed incision, with intra-lenticular instrumentation increasing the risk of a dropped nucleus in the presence of weak zonules or a posterior capsule tear. Femtosecond laser-assisted cataract surgery (FLACS) has been suggested to increase the safety of cataract surgery. ${ }^{8}$ Although the FLACS cohort in this study was smaller, the incidence was comparable to that achieved with standard phacoemulsification. The number of cases of dropped nucleus (three cases among 4,648 procedures) was small, so random effects may influence the incidence. Nonetheless, the choice of operation method by the surgeon may depend on circumstances that we cannot control, which may be a source of bias.

Previous vitrectomy as a risk factor for dropped nucleus was reported by Tajunisah and Reddy. ${ }^{9}$ They also reported hard cataract, polar cataract and high myopia as risk factors. We could not find any relationship with proxies for high myopia in our study (data not shown), and the diagnosis of polar cataract is not included in the list of parameters in EUREQUO. Pseudoexfoliation is a well-known risk factor for capsule complications. ${ }^{10}$ In our study we also found a significant association between pseudoexfoliation and dropped nucleus. This finding, however, must be interpreted with caution as we suspect an underreporting of pseudoexfoliation in the registry. We found a higher frequency of dropped nucleus for medical trainees than for experienced surgeons. Clarke et al. ${ }^{11}$ likewise reported a higher 
frequency of dropped nucleus for trainees, but this complication declined as trainees progressed in training. However, our data do not include information on trainees' years of education.

An eye with lens material in the vitreous is susceptible to postoperative complications including inflammation, increased IOP, and cystoid macular oedema. Such eyes can also develop retinal detachment and venous occlusive disease, putting them at risk for poor functional outcomes. In our study, the outcomes of cataract surgery with a dropped nucleus complication were indeed worse than surgeries with no such complication. However, as can be seen in Table 6 , a considerable number of eyes improved their visual acuity to better functional groups and therefore more useful vision in daily life. The presence of preoperative risk factors such as white cataract or previous vitrectomy implies a worse visual and refractive outcome after dropped nucleus compared with uncomplicated cases (Table 5). Among the poorer visual function groups after surgery, the prevalence of these two risk factors was higher compared with the better visual function groups (data not shown). A weakness in our study is the fact that most of the registry data are self-reported by participating units and surgeons. There is always a risk for underreporting of complications. One strength is the large number of cases across several countries, which makes the statistical analyses more robust and generalizable and the analysis of trends more reliable.

In conclusion, our study shows that there are several risk factors for dropped nucleus; that the incidence of this complication is declining over time; and that the visual and refractive outcomes are not as good as for corresponding cases without the complication of a dropped nucleus. 


\section{References}

1. Lundström M, Frilling E, Montan P. Risk factors for endophthalmitis after cataract surgery - predictors for causative organisms and visual outcome. J Cataract Refract Surg. $2015 ; 41: 2410-2416$.

2. Manning S, Barry P, Henry Y, Rosen P, Stenevi U, Lundström M. Outcome of cataract surgery in post-corneal refractive surgery patients; Study from the European Registry of Quality Outcomes for Cataract and Refractive Surgery. J Cataract Refract Surg. $2015 ; 41: 2358-2365$.

3. Lundström M, Barry P, Brocato L, Fitzpatrick C, Henry Y, Rosen P, Stenevi U. European registry for quality improvement in cataract surgery. Int J Health Care Qual Assur. 2014;27(2):140-151.

4. Lundström M, Dickman M, Henry Y, Manning S, Rosen P, Tassignon MJ, Young D, Stenevi U. Femtosecond laser-assisted cataract surgeries (FLACS) reported to the European Registry of Quality Outcomes for Cataract and Refractive Surgery (EUREQUO): baseline characteristics, surgical procedure, and outcomes. J Cataract Refract Surg 2017; 43:1549-1556.

5. Clark A, Morlet N, Ng JQ, Preen DB, Semmens JB. Whole population trends in complication of cataract surgery over 22 years in Western Australia. Ophthalmology 2011;118:1055-1066.

6. Stilma JS, van der Sluijs FA, van Meurs JC, Mertens DA. Occurrence of retained lens fragments after phacoemulsification in The Netherlands. J Cataract Refract Surg. 1997;23:1177-1182.

7. Aasuri MK, Viswanadh B, Kompella MS, Ajit BM. Risk factors for and management of dropped nucleus during phacoemulsification. J Cataract Refract Surg 2001;27:1428-1432. 
8. Ibrahim T, Goernert P, Rocha G. Intraoperative outcomes and safety of femtosecond laser-assisted cataract surgery: Canadian perspective. Can J Ophthalmol. 2019;54:130135.

9. Tajunisah I, Reddy SC. Dropped nucleus following phacoemulsification cataract surgery. Med J Malaysia 2007;62:364-367.

10. Hemalatha BC, Shetty SB. Analysis of intraoperative and postoperative complications in pseudoexfoliation eyes undergoing cataract surgery. J Clin Diagn Res 2016;10:NC05-8.

11. Clarke C, Ali SF, Murri M, Patel SN, Wang L, Tuft M, Weikert MP, Al-Mohtaseb Z. Outcomes and complication rates of primary resident-performed cataract surgeris at a large tertiary-care county hospital. J Cataract Refract Surg. 2017;43:1563-1570.

\section{Acknowledgement}

All cataract surgeons and clinics who contributed with their data.

What was known before

It has been reported that previous vitrectomy and inexperienced surgeon are risk factors for dropped nucleus. It has also been observed that this complication has decreased in frequency over time.

What this paper adds

There are other important risk factors for dropped nucleus, such as white cataract, small pupil, diabetic retinopathy, pseudoexfoliation, and poor preoperative visual acuity. The visual and refractive outcomes are poorer than for uncomplicated cases, and the outcome partly depends on the existing risk factors. 
Table 1. Number of reported cataract extractions with dropped nucleus as a complication parameter and the number of cases with dropped nucleus in the study period 1 January 2008 to 31 December 2018.

\begin{tabular}{|c|c|c|c|c|c|c|c|c|c|c|c|}
\hline Year & 2008 & 2009 & 2010 & 2011 & 2012 & 2013 & 2014 & 2015 & 2016 & 2017 & 2018 \\
\hline $\begin{array}{l}\text { Number of } \\
\text { reported cataract } \\
\text { extractions } \\
\text { including the } \\
\text { dropped nucleus } \\
\text { parameter }\end{array}$ & 93,344 & 120,186 & 142,594 & 155,717 & 166,189 & 173,118 & 189,682 & 149,143 & 163,482 & 182,560 & 179,333 \\
\hline $\begin{array}{l}\text { Number of } \\
\text { reported dropped } \\
\text { nucleus }\end{array}$ & 87 & 124 & 126 & 145 & 130 & 129 & 162 & 96 & 72 & 85 & 65 \\
\hline Frequency, \% & 0.093 & 0.103 & 0.088 & 0.093 & 0.078 & 0.075 & 0.085 & 0.064 & 0.044 & 0.047 & 0.036 \\
\hline
\end{tabular}


Table 2. Number of reported cases of dropped nucleus per type of surgery (missing data about type of surgery: 4,461 ).

\begin{tabular}{|l|c|c|}
\hline Type of surgery & $\begin{array}{c}\text { Number of } \\
\text { operations }\end{array}$ & $\begin{array}{c}\text { Number of reported cases } \\
\text { with dropped nucleus (\%) }\end{array}$ \\
\hline Phacoemulsification & $1,687,635$ & $1,199(0.071)$ \\
\hline Extracapsular cataract extraction & 14,382 & $8(0.056)$ \\
\hline Phacoemulsification + filtering procedure & 4,212 & $6(0.142)$ \\
\hline Femtosecond laser-assisted cataract extraction & 4,658 & $3(0.064)$ \\
\hline
\end{tabular}


Table 3. Parameters significantly related to dropped nucleus in the univariate regression analysis.

\begin{tabular}{|c|c|c|c|c|c|c|}
\hline Variable & $\mathrm{B}$ & SE & P-value & $\operatorname{Exp}(B)$ & $95 \% \mathrm{Cl}$, lower & $95 \% \mathrm{Cl}$, upper \\
\hline "White cataract" & 1.984 & 0.085 & $<0.001$ & 7.270 & 6.152 & 8.592 \\
\hline Previous vitrectomy & 1.789 & 0.117 & $<0.001$ & 5.981 & 4.753 & 7.526 \\
\hline Preoperative visual acuity, logMAR & 1.393 & 0.086 & $<0.001$ & 4.026 & 3.399 & 4.768 \\
\hline Small pupil & 1.130 & 0.107 & $<0.001$ & 3.094 & 2.507 & 3.820 \\
\hline Diabetic retinopathy & 1.006 & 0.106 & $<0.001$ & 2.735 & 2.220 & 3.369 \\
\hline Pseudoexfoliation & 0.879 & 0.029 & $<0.001$ & 2.408 & 1.564 & 3.708 \\
\hline Glaucoma & 0.303 & 0.104 & 0.004 & 1.354 & 1.105 & 1.660 \\
\hline
\end{tabular}

$95 \% \mathrm{Cl}=95 \%$ confidence interval; $\operatorname{Exp}(\mathrm{B})=$ exponentiation of the $\mathrm{B}$ coefficient; logMAR = logarithm of minimum angle of resolution; $\mathrm{SE}=$ standard error. 
Table 4. Parameters associated with dropped nucleus determined by multivariate analysis (stepwise logistic regression). Dependent variable: dropped nucleus; independent variables: age, gender, preoperative visual acuity (LogMAR), previous vitrectomy, small pupil, white cataract, pseudoexfoliation, diabetic retinopathy, glaucoma, and year of surgery (age and glaucoma not in the equation as not significant).

\begin{tabular}{|l|l|l|l|l|l|l|}
\hline Variable & $\mathrm{B}$ & $\mathrm{SE}$ & $\mathrm{P}$-value & $\mathrm{Exp}(\mathrm{B})$ & $95 \% \mathrm{Cl}$, lower & $95 \% \mathrm{Cl}$, upper \\
\hline "White cataract" & 1.465 & 0.119 & $<0.001$ & 4.329 & 3.428 & 5.466 \\
\hline Previous vitrectomy & 1.379 & 0.145 & $<0.001$ & 3.970 & 2.986 & 5.279 \\
\hline Preoperative visual acuity, logMAR & 1.013 & 0.093 & $<0.001$ & 2.754 & 2.294 & 3.305 \\
\hline Small pupil & 0.849 & 0.127 & $<0.001$ & 2.338 & 1.822 & 3.000 \\
\hline Pseudoexfoliation & 0.593 & 0.263 & 0.024 & 1.810 & 1.081 & 3.031 \\
\hline Diabetic retinopathy & 0.540 & 0.130 & $<0.001$ & 1.716 & 1.329 & 2.215 \\
\hline Gender & 0.441 & 0.064 & $<0.001$ & 1.554 & 1.371 & 1.762 \\
\hline Year of surgery & -0.083 & 0.011 & $<0.001$ & 0.921 & 0.902 & 0.940 \\
\hline
\end{tabular}

$95 \% \mathrm{Cl}=95 \%$ confidence interval; $\operatorname{Exp}(\mathrm{B})=$ exponentiation of the $\mathrm{B}$ coefficient; logMAR = logarithm of minimum angle of resolution; $\mathrm{SE}=$ standard error. 
Table 5. Visual and refractive outcomes of eyes with risk factors, and with or without dropped nucleus complication.

\begin{tabular}{|c|c|c|c|c|c|}
\hline Risk factor & Complication & $\begin{array}{c}\text { Number with the } \\
\text { given } \\
\text { postoperative } \\
\text { visual acuity }\end{array}$ & $\begin{array}{c}\text { Postoperative visual } \\
\text { acuity, mean logMAR } \pm \text { SD } \\
\text { [Decimal notation within } \\
\text { brackets] }\end{array}$ & $\begin{array}{c}\text { Number with the } \\
\text { given } \\
\text { postoperative } \\
\text { refraction }\end{array}$ & $\begin{array}{c}\text { Biometry prediction } \\
\text { within 0.5 D, in per } \\
\text { cent }\end{array}$ \\
\hline White cataract & Dropped nucleus & 116 & $0.32 \pm 0.35$ [ Decimal 0.5] & 129 & 45.0 \\
\hline White cataract & No dropped nucleus & 24,192 & $0.16 \pm 0.29$ [Decimal 0.7] & 24,884 & 61.6 \\
\hline Previous vitrectomy & Dropped nucleus & 60 & $0.39 \pm 0.38$ [Decimal 0.4] & 60 & 45.0 \\
\hline Previous vitrectomy & No dropped nucleus & 15,745 & $0.18 \pm 0.28$ [Decimal 0.7] & 15,950 & 68.3 \\
\hline Small pupil & Dropped nucleus & 76 & $0.25 \pm 0.27$ [Decimal 0.6] & 80 & 58.8 \\
\hline Small pupil & No dropped nucleus & 41,104 & $0.08 \pm 0.21$ [Decimal 0.8] & 41,405 & 69.5 \\
\hline Diabetic retinopathy & Dropped nucleus & 39 & $0.35 \pm 0.35$ [Decimal 0.4] & 42 & 45.2 \\
\hline Diabetic retinopathy & No dropped nucleus & 34,067 & $0.15 \pm 0.24$ [Decimal 0.7] & 34,356 & 16 \\
\hline Pseudoexfoliation & Dropped nucleus & 15 & $0.17 \pm 0.24$ [Decimal 0.7] & 67.6 \\
\hline Pseudoexfoliation & No dropped nucleus & 8,215 & $0.08 \pm 0.20$ [Decimal 0.8] & 8,248 & 62.5 \\
\hline
\end{tabular}

$\operatorname{logMAR}=$ logarithm of minimum angle of resolution; $\mathrm{SD}=$ standard deviation. 
Table 6. Change in visual acuity groups* for patients with a dropped nucleus complication. Details are given for 619 cases with full follow-up data.

\begin{tabular}{|c|c|c|c|c|c|c|}
\hline $\begin{array}{l}\text { Preoperative visual } \\
\text { acuity groups }\end{array}$ & $\begin{array}{l}\text { Number of } \\
\text { cases }\end{array}$ & $\begin{array}{l}\text { Postoperative visual } \\
\text { acuity group }\end{array}$ & $\begin{array}{l}\text { Postoperative } \\
\text { visual acuity group }\end{array}$ & $\begin{array}{l}\text { Postoperative visual } \\
\text { acuity group }\end{array}$ & $\begin{array}{l}\text { Postoperative visual } \\
\text { acuity group }\end{array}$ & $\begin{array}{l}\text { Postoperative } \\
\text { visual acuity group }\end{array}$ \\
\hline & & $\begin{array}{c}1(\log M A R-0.2-0.0), \\
\text { [Decimal } 1.6 \text { to } 1.0] \\
n(\%)\end{array}$ & $\begin{array}{c}2(\log M A R \quad 0.1) \\
\text { [Decimal } 0.8] \\
n(\%)\end{array}$ & $\begin{array}{c}3 \text { (logMAR } 0.2-0.3), \\
\text { [Decimal } 0.6 \text { to } 0.5] \\
n(\%)\end{array}$ & $\begin{array}{c}4 \text { (logMAR 0.4-0.5), } \\
\text { [Decimal } 0.4 \text { to } 0.3] \\
n(\%)\end{array}$ & $\begin{array}{c}5 \text { (logMAR } 0.6 \text { or } \\
\text { worse), [Decimal } \\
0.25 \text { or worse] } \\
\text { n (\%) }\end{array}$ \\
\hline $\begin{array}{l}1(\log M A R-0.2-0.0) \\
{[\text { Decimal } 1.6 \text { to } 1.0]}\end{array}$ & 12 & 7 (58.3) & 0 & $3(25.0)$ & $1(8.3)$ & $1(8.3)$ \\
\hline $\begin{array}{l}2(\log M A R 0.1) \\
{[\text { Decimal } 0.8]}\end{array}$ & 36 & $14(38.9)$ & $8(22.2)$ & $8(22.2)$ & $3(8.3)$ & $3(8.3)$ \\
\hline $\begin{array}{l}3(\log M A R 0.2-0.3) \\
\text { [Decimal } 0.6 \text { to } 0.5 \text { ] }\end{array}$ & 236 & $94(39.8)$ & $56(23.7)$ & $59(25.0)$ & $16(6.8)$ & $11(4.7)$ \\
\hline $\begin{array}{l}4(\log M A R 0.4-0.5) \\
\text { [Decimal } 0.4 \text { to } 0.3 \text { ] }\end{array}$ & 146 & $55(37.7)$ & $32(21.9)$ & $33(22.6)$ & $15(10.3)$ & $11(7.5)$ \\
\hline $\begin{array}{l}5 \text { (logMAR } 0.6 \text { or worse) } \\
\text { [Decimal } 0.25 \text { or worse] }\end{array}$ & 189 & $54(28.6)$ & $20(10.6)$ & $44(23.3)$ & 22 (11.6) & 49 (25.9) \\
\hline All & 619 & $224(36.2)$ & $116(18.8)$ & $147(23.7)$ & $57(9.2)$ & 75 (12.1) \\
\hline
\end{tabular}

*according to Lundström et al. ${ }^{4}$

Green text: improvement to a better visual acuity group; red text: deterioration to a worse visual acuity group.

logMAR = logarithm of minimum angle of resolution

Decimal notation within brackets 